The 118 papers in parts I and II deal with all the analysis techniques which are normally associated with SEM and STEM (X-rays, Auger electrons, electron energy loss, cathodoluminescence, electron diffraction) and also microscopic techniques like SIMS, LAMMA, PIXE and soft $X$-ray contact microscopy which enjoy association with SEM while they do not employ scanning electron beams.

The papers in part 1 deal, amongst others, with subjects of imaging, contamination, electron guns, automation and the structure of conductive coatings, which is very relevant in high-resolution SEM. Useful hints on the application of convergent-beam diffraction are also presented. Environment and forensic (in the wide sense) applications are well represented. The analysis of radioactive particulates from a disintegrated Russian satellite represents an interesting example of both categories.

The growing interest of biological sciences (including biomedical) in SEM and associated microscopic techniques is again evident from the number of papers. Two parts of the 1980 proceedings are devoted to biological applications and the pace is set by the keynote paper on biophysical applications of SEM. The application of the newer techniques like ELS and SIMS in biology is dealt with in review papers.

Botanists can find an extensive bibliography on microanalysis in botany. Laser microscopy and microanalysis find extensive application in biology. Applications of soft $X$-ray microscopy and the interesting imaging technique using total $X$-ray signals are also reported by biologists.

The discussions with reviewers which are included with each paper not only contribute significantly to the amount of useful information contained in the papers but also serve as additional sources of reference since a list containing names and addresses of reviewers is included in each part.

The editors succeeded in maintaining a balance between applications, tutorials and review papers which make these books valuable assets for all SEM users. The SEM Inc. conferences are recognized as one of the foremost forums for the reporting of developments and applications in SEM and associated techniques and the 1980 proceedings should take their place as valuable additions to an accepted journal.

J. SMUTS

Materials Research Division

Iscor

PO Box 450

Pretoria 0001

Republic of South Africa
J. Appl. Cryst. (1982). 15, 140-141

\section{Characterisation of crystal growth defects by X-ray methods. NATO Advanced Study Institutes Series, Series B: Physics. Edited by B. K. Tanner and D.K. Bowen.} Pp. 589. New York: Plenum Press, 1980. Price US $\$ 65.00$.

Single crystals play a very important role in modern industry, which needs highly perfect single crystals of a variety of different materials. As a consequence, the interest in techniques for analysing crystal perfection has increased. Among the more sophisticated of these techniques are $X$-ray methods, and numerous papers and books are being published on this subject. But the book under review has an advantage over others because of the wide range that it covers.

This book is the proceedings of a NATO Institute, held in 1979, in Durham, England. The authors include wellknown scientists doing research in crystal growth and X-ray topography. It consists of 25 chapters and is supplied with seven appendices.

Many problems on growth defects and methods of their detection are discussed. The main points for consideration in this book may be divided into two parts.

Firstly, there is a survey of various types of growth defects, the mechanisms of their generation, both in metal (G. Champier) and in non-metal (H. Klapper) crystals, growing by various methods; the problems connected with the industrial applications of crystals (E. S. Meieran); and crystal defect problems from the point of view of the device operation (charge-coupled devices, magnetic bubble memories, surface acoustic wave devices) (A. D. Milne).

The main part of the book is devoted to the investigation of crystal defects by $X$ ray methods. Emphasis is given to $X$-ray topography. First of all it is necessary to remark on the clear presentation of the physical basis of the detection of crystal defects by means of X-ray topography: elementary dynamical theory of X-ray diffraction (M. Hart) and analysis of the diffraction phenomena in crystals of various degrees of perfection (N. Kato). A detailed review of transmission $X$-ray topography is presented (A. R. Lang, J. Chikawa). The basic techniques are discussed: section topography, projection topography, the stereo method, the weak-beam method, and the kinematic image technique for the detection of small defects. The interpretation of X-ray topographs and analysis of different types of defects are described in papers by J. R. Patel, A. R. Lang and J. Chikawa: line and planar defects, precipitations and inclusions.

By contrast, reflection topography is not represented in such detail. Armstrong's paper deals with diffraction contrast and the application of a stereographic projection description for X-ray reflection topography. The panel discussion on reflection topography is reviewed by R. W. Armstrong. Researchers active in this field discuss image contrast in this method, some problems of the double-crystal reflection method and the Berg-Barrett method.

The experimental technique for $X$-ray topography: X-ray sources (U. Bonse), $X$ ray detectors (A. R. Lang), sample preparation (D. K. Bowen); is presented in detail. Tables for practical use list characteristic $X$-ray tubes and storage rings suited for $X$-ray generation. Synchrotron $X$-ray sources are widely used today. The use of white-beam synchrotron radiation (J.Miltat) and also monochromatic synchrotron radiation (M. Sauvage) for X-ray topography, as well as problems of control of wavelengths, polarization, time structure and divergence for synchrotron radiation topography ( $M$. Hart), are discussed. Emphasis is laid on the advantage of synchrotron radiation for dynamic and in situ experiments (B. K. Tanner). The use of TV systems for $X$-ray topography is noted ( $J$. Chikawa) and a real-time $X$ ray topography system for observing the dynamics of defects and strain fields, with a spatial resolution down to $10 \mu \mathrm{m}$ and a time resolution down to $20 \mathrm{~ms}$, are described (W. Hartmann). The application of synchrotron radiation for absorption microscopy is discussed by D. K. Bowen.

The book also examines the investigation of diffuse $X$-ray scattering due to point defects (J.R. Patel \& J.R. Schneider) and the application of $i$-ray diffractometry (J. R. Schneider).

A very useful purpose is served by the paper by M. Hart on the costs of X-ray topography: experimental arrangements for transmission topography and doublecrystal methods (X-ray sources, cameras, detectors, commercial apparatus, as well as apparatus built in laboratories) and their cost are described.

The useful exercises in X-ray topography will help students to master the experimental procedures and interpretation of $X$-ray topographs. There are many excellent $X$-ray pictures of growth defects in different crystals.

This book is a good guide to the X-ray topographic investigation of crystal defects today and, as such, can be recommended to anyone interested in crystal growth or X-ray topography, and to those who use crystals in various devices. The clear manner of presentation of the 
dynamical theory of X-ray diffraction and the large number of practical applications makes the book very useful for newcomers to the field of X-ray topography.

\section{E. P. KOSTYUKOVA}

Machinery.Institute

Academy of Sciences of the USSR

Griboyedov Street 4

Moscow 101830

USSR

\section{J. Appl. Cryst. (1982). 15, 141-142}

\section{Current topics in materials sci-} ence, Vols. 5 and 6 . Edited by E. Kaldis. Vol. 5: pp. vi +617 ; Vol. 6: pp. vii + 481. Amsterdam: North-Holland, 1980. Price: Vol. 5 , US $\$ 87.75$; Vol. 6 , US $\$ 97.50$.

These two volumes are more-or-less concerned with electronic materials. Therefore, they are reviewed together in one single article from the standpoint of solid-state electronics.

In solid-state electronics, the improvements or the new functions of electronic device characteristics are driven forward by refinements of the present materials or developments of new electronic materials, respectively. This being so, electronic materials engineers must always challenge the development of new materials for electronic use, giving attention to the physical properties, the crystal growth, the reaction with the other materials, and so on.

These two volumes seem to present the answers to many questions asked by the electronic-materials engineers. Generally speaking, the essential points of materials research for electronics are described under a good combination of selected processes and selected materials, though a wide range of subject matters is put in order. Ten articles in all are reviewed one after another as follows.

The first two reviews of Vol. 5 , that is The preparation of gallium arsenide, by L. Hollan \& J. P. Hallais, and Application of the theory of rate processes in the CVD of GaAs, by R. Cardoret, are focused on GaAs and related compounds, which are very important materials for the future of solid-state electronics. The following are some of the reasons for this: (1) In the field of integrated circuits, the higher mobility of the electrons in GaAs leads to faster circuits compared with $\mathrm{Si}$; (2) GaAs has a direct bandgap which makes it suitable for integrated-optic devices including light emitters, receivers and guide; (3) As seen in the study of the GaAs-AIAs superlattice, the series of these compounds is expected to have new physical properties which may lead to new functional devices. GaAs crystals have an advantage in the ease of obtaining semiinsulating materials as a substrate for thin-film integrated circuits, whilst with $\mathrm{Si}$ this cannot be done unless insulating substrates, such as sapphire and spinel, are used. For this to be realized for the above mentioned devices, however, there are many problems on material preparations and processes to be solved.

From these two articles, we can obtain a knowledge of GaAs covering all aspects from the growth of the bulk crystal, liquid-phase and vapour-phase epitaxy (including MOCVD), molecular beam epitaxy, characterizations, and materials properties, to applications. Following this, the theoretical considerations of GaAs CVD processes are treated in regard to two kinds of growth, that is growth by layers and direct or continuous growth, referring to the recent fundamental investigations of the transition between these two growth modes by statistical methods and by simulation on computers.

A. A. Chernov \& V. V. Sipyagin review Peculiarities in crystal growth from aqueous solutions connected with their structures. The relationship between the aggregating state in a liquid phase and the perfection of the grown crystal is a subject still to be made clear. Progress in this field will greatly contribute to solidstate electronics in the matter of obtaining more-perfect crystals from aqueous solutions. The authors of this article attempt to summarize this important problem by referring to associations in aqueous solutions and crystal growth, anomalous temperature dependence of growth rates, investigation of films of solution by NMR methods and temperature dependence of viscosity (although electronic materials, in the narrow sense, are not involved here).

The following chapter, written by H. K. Cammenga, is devoted to Evaporation mechanism of liquids. This article is mainly concerned with the theoretical and the experimental aspects of liquid evaporation and condensation at solid surfaces. In the field of electronic materials, there are many opportunities to meet with exchange phenomena. That is, in the chemical vapour deposition, including epitaxial growth, many kinds of liquids are utilized as source materials, which are evaporated directly or bubbled to transport material to the crystal wafer surface. From this point of view, it is valuable for the electronic-materials engineer to understand the thermodynamics and the kinetics of these phenomena fundamentally, as touched on in this article.
The final chapter of Vol. 5, by A. Baronnet, discusses Polytypism in micas: a survey with emphasis on the crystal growth aspect. Micas have been utilized for electronic materials from the old days and they have excellent electrical characteristics as many kinds of capacitors in the respects of long life, high breakdown voltage, and insensitivity to temperature variations. On the other hand, polytypism is also observed in other crystals, such as SiC, ZnS, and so on. From the standpoint of electronic materials, there are two points to emphasize in regard to polytypism. One of them is that polytypism obstructs the perfection of single crystals by acting as a kind of physical contamination. The other, however, is that, if the polytypism can be controlled artificially and precisely, new electronic materials may be prepared. An example is given by Dr L. Esaki of IBM, in the GaAs-AIAs superlattice. The reason for the appearance of huge periodicities in natural crystals is one of the most prominent questions generated by the polytypism phenomenon. This chapter reviews what is known about polytypism in micas: (1) by showing in detail how complex the structural aspect of this phenomenon is; (2) by discussing the stability of polytypes; and (3) by focusing attention on the relationship between crystal growth conditions and polytypism. These three aspects are also common to other polytypic crystals.

The first chapter of Vol. 6 presents The characterization of semiconductor materials and structures using electrochemical techniques, by M. M. Factor et al. The subjects of metal or semiconductor electrodes, electrical characterization of GaAs and other semiconductors, microstructural evaluation of semiconductor materials by electrochemical dissolution, and, additionally, further exploitation of electrochemical techniques in the analysis and fabrication of semiconductor materials and devices are concisely reviewed. Modern electronic devices using semiconductor materials are increasingly surface- and interface-layers dominated. The cleanliness and perfection of these layers is becoming more and more important for the fabrication of the devices. We must reveal, as the first step, the microinhomogeneities and the microstructural defects in these layers. The various electrochemical techniques, as described in this chapter, are indispensable for the characterization of semiconductor materials and devices. The etching method, for instance, is an inverse process to crystal growth and gives us the growth history, which leads to the elucidation of the origin of microdefects.

Properties and behaviour of the zinc oxide/electrolyte phase boundary are re- 